Supplementary Online Material

\title{
A Mechanism for Biologically-Induced Iodine Release from sea-ice
}

\author{
Alfonso Saiz-Lopez and Christopher S. Boxe \\ Earth and Space Science Division, NASA Jet Propulsion Laboratory, California Institute \\ of Technology, Pasadena, California 91109 \\ Correspondence should be addressed to ASL: (Alfonso.Saiz-Lopez@jpl.nasa.gov)
}

\section{This PDF file includes:}

Supporting online text

Tables 1, 2 and 3

References 
Table 1. Gas Phase Reactions and Rate Constants

\begin{tabular}{|c|c|c|c|}
\hline$\#$ & Bimolecular Reactions & Rate Constants & References \\
\hline 1. & $\mathrm{O}\left({ }^{1} \mathrm{D}\right)+\mathrm{N}_{2} \rightarrow \mathrm{O}+\mathrm{N}_{2}$ & $1.8 \times 10^{-11} \mathrm{e}^{(110 / \mathrm{T})}$ & 2 \\
\hline 2. & $\mathrm{O}\left({ }^{1} \mathrm{D}\right)+\mathrm{O}_{2} \rightarrow \mathrm{O}+\mathrm{O}_{2}$ & $3.2 \times 10^{-11} \mathrm{e}^{(70 / \mathrm{T})}$ & 2 \\
\hline 3. & $\mathrm{O}\left({ }^{1} \mathrm{D}\right)+\mathrm{H}_{2} \mathrm{O} \rightarrow \mathrm{OH}+\mathrm{OH}$ & $2.2 \times 10^{-10}$ & 2 \\
\hline 4. & $\mathrm{O}\left({ }^{1} \mathrm{D}\right)+\mathrm{CH}_{4} \rightarrow \mathrm{CH}_{3}+\mathrm{OH}(0.75), \mathrm{CH}_{3} \mathrm{O}+\mathrm{H}(0.2), \mathrm{HCHO}+\mathrm{H}_{2}(0.05)$ & $1.5 \times 10^{-10}$ & 2 \\
\hline 5. & $\mathrm{O}\left({ }^{1} \mathrm{D}\right)+\mathrm{H}_{2} \rightarrow \mathrm{OH}+\mathrm{H}$ & $1.1 \times 10^{-10}$ & 2 \\
\hline 6. & $\mathrm{OH}+\mathrm{CO} \rightarrow \mathrm{H}+\mathrm{CO}_{2}$ & $1.5 \times 10^{-13} \times\left(1+0.6 \times P_{a t m}\right)$ & 2 \\
\hline 7. & $\mathrm{HO}_{2}+\mathrm{NO} \rightarrow \mathrm{NO}_{2}+\mathrm{OH}$ & $3.5 \times 10^{-12} \mathrm{e}^{(250 / \mathrm{T})}$ & 2 \\
\hline 8. & $\mathrm{O}_{3}+\mathrm{HO}_{2} \rightarrow \mathrm{OH}+2 \mathrm{O}_{2}$ & $1.1 \times 10^{-14} \mathrm{e}^{(-500 / \mathrm{T})}$ & 2 \\
\hline 9. & $\mathrm{HO}_{2}+\mathrm{HO}_{2} \rightarrow \mathrm{H}_{2} \mathrm{O}_{2}+\mathrm{O}_{2}$ & $2.3 \times 10^{-13} \mathrm{e}^{(600 / \mathrm{T})}$ & 2 \\
\hline 10. & $\mathrm{OH}+\mathrm{H}_{2} \rightarrow \mathrm{H}_{2} \mathrm{O}+\mathrm{H}$ & $5.5 \times 10^{-12} \mathrm{e}^{(-2000 / \mathrm{T})}$ & 2 \\
\hline 11. & $\mathrm{O}_{3}+\mathrm{OH} \rightarrow \mathrm{HO}_{2}+\mathrm{O}_{2}$ & $1.6 \times 10^{-12} \mathrm{e}^{(-940 / \mathrm{T})}$ & 2 \\
\hline \multirow[t]{4}{*}{12.} & $\mathrm{OH}+\mathrm{HNO}_{3} \rightarrow \mathrm{H}_{2} \mathrm{O}+\mathrm{NO}_{3}$ & $\mathrm{k}_{0}=7.2 \times 10^{-15} \mathrm{e}^{(785 / \mathrm{T})}$ & 2 \\
\hline & & $\mathrm{k}_{2}=4.1 \times 10^{-16} \mathrm{e}^{(1440 / \mathrm{T})}$ & \\
\hline & & $\mathrm{k}_{3}=1.9 \times 10^{-33} \mathrm{e}^{(725 / \mathrm{T})}$ & \\
\hline & & $\mathrm{k}=\mathrm{k}_{0}+\left(\mathrm{k}_{3} \times[\mathrm{M}] /\left(1+\mathrm{k}_{3} \times[\mathrm{M}] / \mathrm{k}_{2}\right)\right)$ & \\
\hline 13. & $\mathrm{H}_{2} \mathrm{O}_{2}+\mathrm{OH} \rightarrow \mathrm{H}_{2} \mathrm{O}+\mathrm{HO}_{2}$ & $2.9 \times 10^{-12} \mathrm{e}^{(-160 / \mathrm{T})}$ & 2 \\
\hline 14. & $\mathrm{OH}+\mathrm{HO}_{2} \mathrm{NO}_{2} \rightarrow \mathrm{NO}_{2}+\mathrm{HO}_{2}+\mathrm{OH}$ & $1.3 \times 10^{-12} \mathrm{e}^{(380 / \mathrm{T})}$ & 2 \\
\hline 15. & $\mathrm{OH}+\mathrm{HO}_{2} \rightarrow \mathrm{H}_{2} \mathrm{O}+\mathrm{O}_{2}$ & $4.8 \times 10^{-11} \mathrm{e}^{(250 / \mathrm{T})}$ & 2 \\
\hline 16. & $\mathrm{OH}+\mathrm{HONO} \rightarrow \mathrm{H}_{2} \mathrm{O}+\mathrm{NO}_{2}$ & $1.8 \times 10^{-11} \mathrm{e}^{(390 / \mathrm{T})}$ & 2 \\
\hline 17. & $\mathrm{C}_{2} \mathrm{H}_{5}+\mathrm{O}_{2} \rightarrow \mathrm{C}_{2} \mathrm{H}_{4}+\mathrm{HO}_{2}$ & $2 \times 10^{-14}$ & $2, \mathrm{~b}$ \\
\hline 18. & $\mathrm{OH}+\mathrm{CH}_{4} \rightarrow \mathrm{CH}_{3}+\mathrm{H}_{2} \mathrm{O}$ & $2.45 \times 10^{-12} \mathrm{e}^{(-1775 / \mathrm{T})}$ & 2 \\
\hline 19. & $\mathrm{O}\left({ }^{3} \mathrm{P}\right)+\mathrm{CH}_{3} \rightarrow \mathrm{CH}_{3} \mathrm{O}$ & $1.1 \times 10^{-10}$ & 2 \\
\hline 20. & $\mathrm{CH}_{3} \mathrm{O}_{2}+\mathrm{HO}_{2} \rightarrow \mathrm{CH}_{3} \mathrm{OOH}+\mathrm{O}_{2}$ & $3.8 \times 10^{-13} \mathrm{e}^{(800 / \mathrm{T})}$ & 2 \\
\hline 21. & $\mathrm{CH}_{3} \mathrm{OOH}+\mathrm{OH} \rightarrow \mathrm{CH}_{3}(\mathrm{O}) \mathrm{O}+\mathrm{H}_{2} \mathrm{O}$ & $0.7 \times 3.8 \times 10^{-12} \mathrm{e}^{(200 / \mathrm{T})}$ & 2 \\
\hline 22. & $\mathrm{CH}_{3} \mathrm{O}+\mathrm{O}_{2} \rightarrow \mathrm{CH}_{2} \mathrm{O}+\mathrm{HO}_{2}$ & $3.9 \times 10^{-14} \mathrm{e}^{(-900 / \mathrm{T})}$ & 2 \\
\hline 23. & $\mathrm{OH}+\mathrm{HCHO} \rightarrow \mathrm{H}_{2} \mathrm{O}+\mathrm{HCO}$ & $8.8 \times 10^{-12} \mathrm{e}^{(25 / \mathrm{T})}$ & 2 \\
\hline 24. & $\mathrm{HCO}+\mathrm{O}_{2} \rightarrow \mathrm{CO}+\mathrm{HO}_{2}$ & $3.5 \times 10^{-12} \mathrm{e}^{(140 / \mathrm{T})}$ & 2 \\
\hline 25. & $\mathrm{CH}_{3} \mathrm{O}_{2}+\mathrm{CH}_{3} \mathrm{O}_{2} \rightarrow 2 \mathrm{CH}_{3} \mathrm{O}+\mathrm{O}_{2} 29 \%$ & $0.29 \times 2.5 \times 10^{-13} \mathrm{e}^{(190 / \mathrm{T})}$ & 2 \\
\hline 26. & $\mathrm{NO}+\mathrm{CH}_{3} \mathrm{O}_{2} \rightarrow \mathrm{NO}_{2}+\mathrm{CH}_{3} \mathrm{O}$ & $3 \times 10^{-12} \mathrm{e}^{(280 / \mathrm{T})}$ & 2 \\
\hline 27. & $\mathrm{NO}+\mathrm{O}_{3} \rightarrow \mathrm{NO}_{2}+\mathrm{O}_{2}$ & $2 \times 10^{-12} \mathrm{e}^{(-1400 / \mathrm{T})}$ & 2 \\
\hline 28. & $\mathrm{NO}+\mathrm{NO}_{3} \rightarrow 2 \mathrm{NO}_{2}$ & $1.5 \times 10^{-11} \mathrm{e}^{(170 / \mathrm{T})}$ & 2 \\
\hline 29. & $\mathrm{NO}_{3}+\mathrm{HCHO} \rightarrow$ Products & $5.8 \times 10^{-16}$ & $2, \mathrm{~b}$ \\
\hline 30. & $\mathrm{HO}_{2}+\mathrm{SO}_{2} \rightarrow$ Products & $1 \times 10^{-18}$ & $2, \mathrm{~b}$ \\
\hline 31. & $\mathrm{~N}_{2} \mathrm{O}_{5}+\mathrm{H}_{2} \mathrm{O} \rightarrow 2 \mathrm{HNO}_{3}$ & $2.5 \times 10^{-22}$ & $2, \mathrm{~b}$ \\
\hline 32. & $\mathrm{NO}_{2}+\mathrm{O}_{3} \rightarrow \mathrm{NO}_{3}+\mathrm{O}_{2}$ & $1.2 \times 10^{-13} \mathrm{e}^{(-2450 / \mathrm{T})}$ & 2 \\
\hline 33. & $\mathrm{OH}+\mathrm{O}\left({ }^{3} \mathrm{P}\right) \rightarrow \mathrm{H}+\mathrm{O}_{2}$ & $2.2 \times 10^{-11} \mathrm{e}^{(120 / \mathrm{T})}$ & 2 \\
\hline 34. & $\mathrm{O}\left({ }^{3} \mathrm{P}\right)+\mathrm{HO}_{2} \rightarrow \mathrm{OH}+\mathrm{O}_{2}$ & $3 \times 10^{-11} \mathrm{e}^{(200 / \mathrm{T})}$ & 2 \\
\hline 35. & $\mathrm{H}_{2} \mathrm{O}_{2}+\mathrm{O}\left({ }^{3} \mathrm{P}\right) \rightarrow \mathrm{OH}+\mathrm{HO}_{2}$ & $1.4 \times 10^{-12} \mathrm{e}^{(-2000 / \mathrm{T})}$ & 2 \\
\hline 36. & $\mathrm{OH}+\mathrm{OH} \rightarrow \mathrm{H}_{2} \mathrm{O}+\mathrm{O}\left({ }^{3} \mathrm{P}\right)$ & $4.2 \times 10^{-12} \mathrm{e}^{(-240 / \mathrm{T})}$ & 2 \\
\hline 37. & $\mathrm{O}_{3}+$ Alkenes $\rightarrow$ Products & $1.2 \times 10^{-14} \mathrm{e}^{(-2630 / \mathrm{T})}$ & $2, \mathrm{~b}$ \\
\hline
\end{tabular}




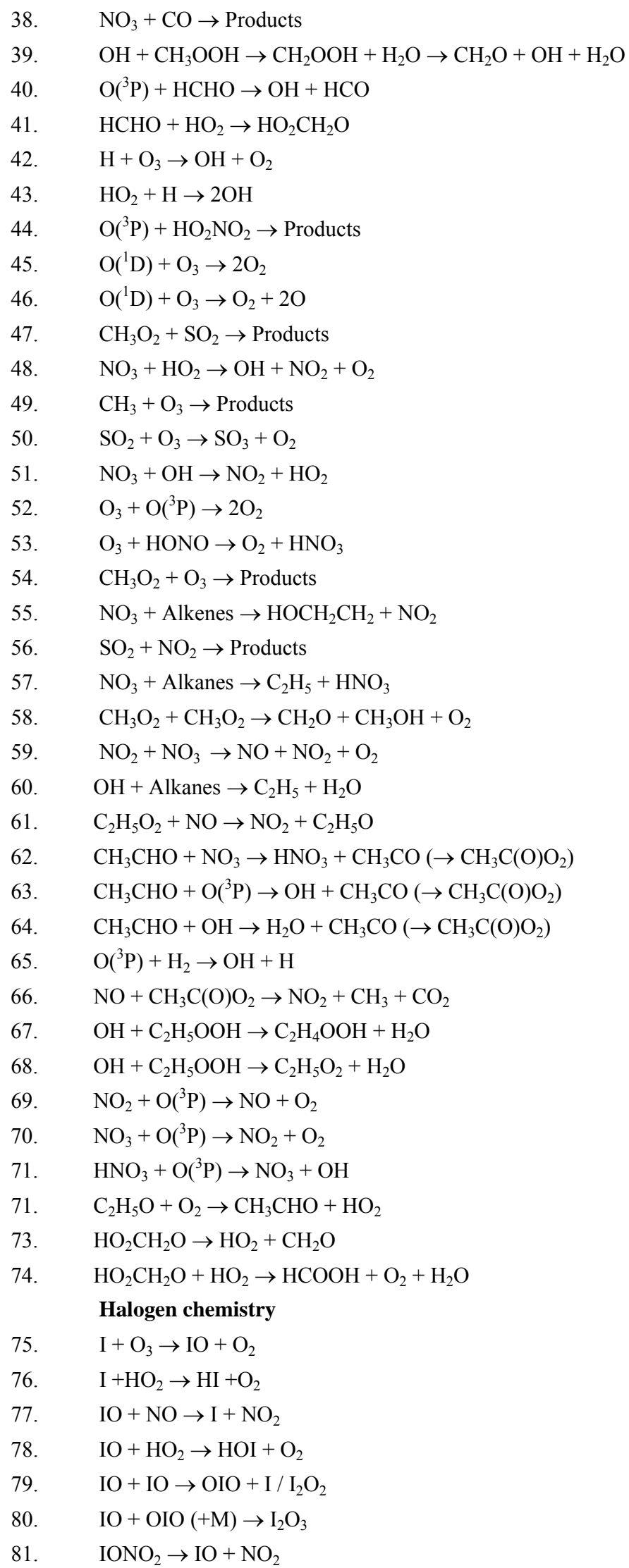

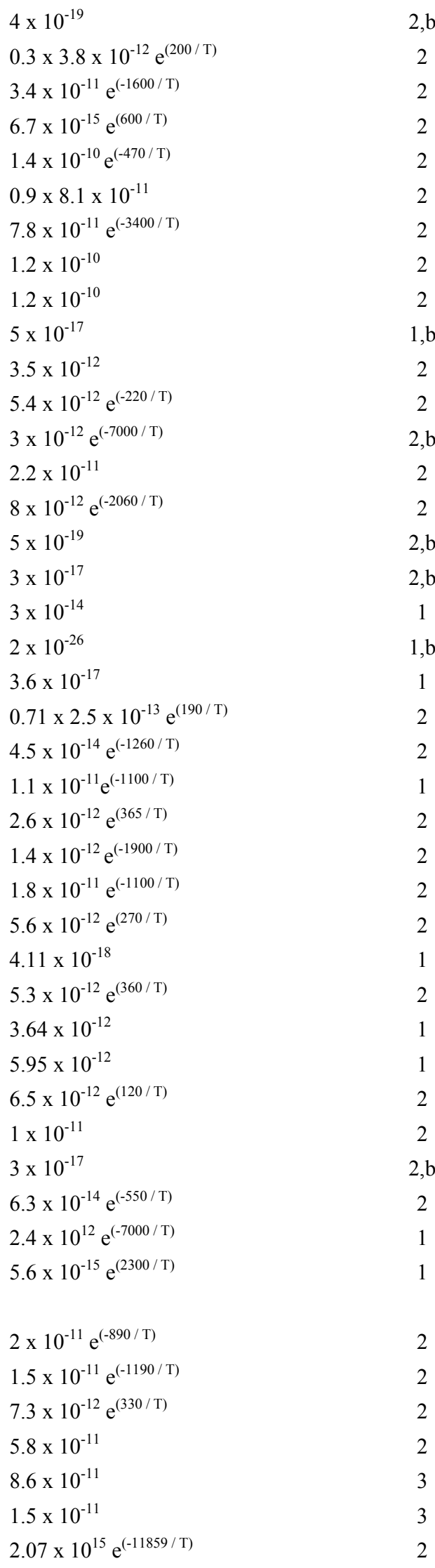




$$
\begin{aligned}
& \text { 82. } \mathrm{OH}+\mathrm{HI} \rightarrow \mathrm{I}+\mathrm{H}_{2} \mathrm{O} \\
& \text { 83. } \mathrm{HOI}+\mathrm{OH} \rightarrow \mathrm{IO}+\mathrm{H}_{2} \mathrm{O} \\
& \text { 84. } \quad \mathrm{IO}+\mathrm{DMS} \rightarrow \text { Products } \\
& \text { 85. } \mathrm{INO}_{2} \rightarrow \mathrm{I}+\mathrm{NO}_{2} \\
& \text { 86. } \mathrm{Br}+\mathrm{O}_{3} \rightarrow \mathrm{BrO}+\mathrm{O}_{2} \\
& \text { 87. } \mathrm{HBr}+\mathrm{OH} \rightarrow \mathrm{Br}+\mathrm{H}_{2} \mathrm{O} \\
& \text { 88. } \mathrm{Br}+\mathrm{HO}_{2} \rightarrow \mathrm{HBr}+\mathrm{O}_{2} \\
& \text { 89. } \mathrm{Br}+\mathrm{HCHO} \rightarrow \mathrm{HBr}+\mathrm{HCO} \\
& \text { 90. } \mathrm{Br}+\mathrm{CH}_{3} \mathrm{CHO} \rightarrow \mathrm{HBr} \mathrm{CH}_{3} \mathrm{CO} \\
& \text { 91. } \mathrm{BrO}+\mathrm{HO}_{2} \rightarrow \mathrm{HOBr}+\mathrm{O}_{2} \\
& \text { 92. } \mathrm{BrO}+\mathrm{NO} \rightarrow \mathrm{Br}+\mathrm{NO}_{2} \\
& \text { 93. } \mathrm{BrO}+\mathrm{CH}_{3} \mathrm{SCH}_{3} \rightarrow \mathrm{CH}_{3} \mathrm{SOCH}_{3}+\mathrm{Br} \\
& \text { 94. } \mathrm{BrO}+\mathrm{BrO} \rightarrow 2 \mathrm{Br}+\mathrm{O}_{2} \\
& \text { 95. } \mathrm{BrO}+\mathrm{BrO} \rightarrow \mathrm{Br}_{2}+\mathrm{O}_{2} \\
& \text { 96. } \mathrm{BrONO}_{2} \rightarrow \mathrm{BrO}+\mathrm{NO}_{2} \\
& \text { 97. } \mathrm{BrO}+\mathrm{IO} \rightarrow \mathrm{Br}+\mathrm{I}+\mathrm{O}_{2} / \mathrm{Br}+\mathrm{OIO} \\
& \text { 98. } \mathrm{Cl}+\mathrm{CH}_{4} \rightarrow \mathrm{HCl}+\mathrm{CH}_{3} \\
& \text { 99. } \mathrm{HCl}+\mathrm{OH} \rightarrow \mathrm{H}_{2} \mathrm{O}+\mathrm{Cl} \\
& \text { 100. } \mathrm{Cl}+\mathrm{O}_{3} \rightarrow \mathrm{ClO}+\mathrm{O}_{2} \\
& \text { 101. } \mathrm{ClO}+\mathrm{HO}_{2} \rightarrow \mathrm{HOCl}+\mathrm{O}_{2} \\
& \text { 102. } \mathrm{ClO}+\mathrm{NO} \rightarrow \mathrm{Cl}+\mathrm{NO}_{2} \\
& \text { 103. } \mathrm{ClO}+\mathrm{IO} \rightarrow 0.2\left(\mathrm{I}+\mathrm{Cl}+\mathrm{O}_{2}\right) \\
& \text { 104. } \mathrm{Cl}+\text { Alkanes } \rightarrow \mathrm{HCl}+\mathrm{CH}_{3} \mathrm{OO} \\
& \text { 105. } \mathrm{Cl}+\text { Alkenes } \rightarrow \mathrm{HCl}+\mathrm{CH}_{3} \mathrm{OO} \\
& \text { 106. } \mathrm{Cl}+\mathrm{HCHO} \rightarrow \mathrm{HCl}+\mathrm{HO}_{2}+\mathrm{CO} \\
& \text { 107. } \mathrm{ClO}+\mathrm{CH}_{3} \mathrm{OO} \rightarrow \mathrm{Cl}+\mathrm{HCHO}+\mathrm{HO}_{2} \\
& \text { 108. } \mathrm{ClO}+\mathrm{ClO} \rightarrow \mathrm{Cl}_{2} \mathrm{O}_{2} \\
& \text { 109. } \mathrm{Cl}_{2} \mathrm{O}_{2} \rightarrow \mathrm{ClO}+\mathrm{ClO} \\
& \text { 110. } \mathrm{ClONO}_{2} \rightarrow \mathrm{ClO}+\mathrm{NO}_{2} \\
& \text { 111. } \mathrm{Cl}+\mathrm{ClONO}_{2} \rightarrow \mathrm{Cl}_{2}+\mathrm{NO}_{3} \\
& \text { 112. } \mathrm{Cl}+\mathrm{H}_{2} \mathrm{O}_{2} \rightarrow \mathrm{HCl}+\mathrm{HO}_{2} \\
& \text { 113. } \mathrm{Br}_{2}+\mathrm{Cl} \rightarrow \mathrm{BrCl}+\mathrm{Br} \\
& \text { 114. } \mathrm{BrCl}+\mathrm{Br} \rightarrow \mathrm{Br}_{2}+\mathrm{Cl} \\
& \text { 115. } \mathrm{Cl}_{2}+\mathrm{Br} \rightarrow \mathrm{BrCl}+\mathrm{Cl} \\
& \text { 116. } \mathrm{BrCl}+\mathrm{Cl} \rightarrow \mathrm{Cl}_{2}+\mathrm{Br} \\
& \text { 117. } \mathrm{ClO}+\mathrm{BrO} \rightarrow \mathrm{Br}+\mathrm{OClO} \\
& \text { 118. } \mathrm{ClO}+\mathrm{BrO} \rightarrow \mathrm{Br}+\mathrm{Cl}+\mathrm{O}_{2} \\
& \text { 119. } \mathrm{ClO}+\mathrm{BrO} \rightarrow \mathrm{BrCl}+\mathrm{O}_{2}
\end{aligned}
$$

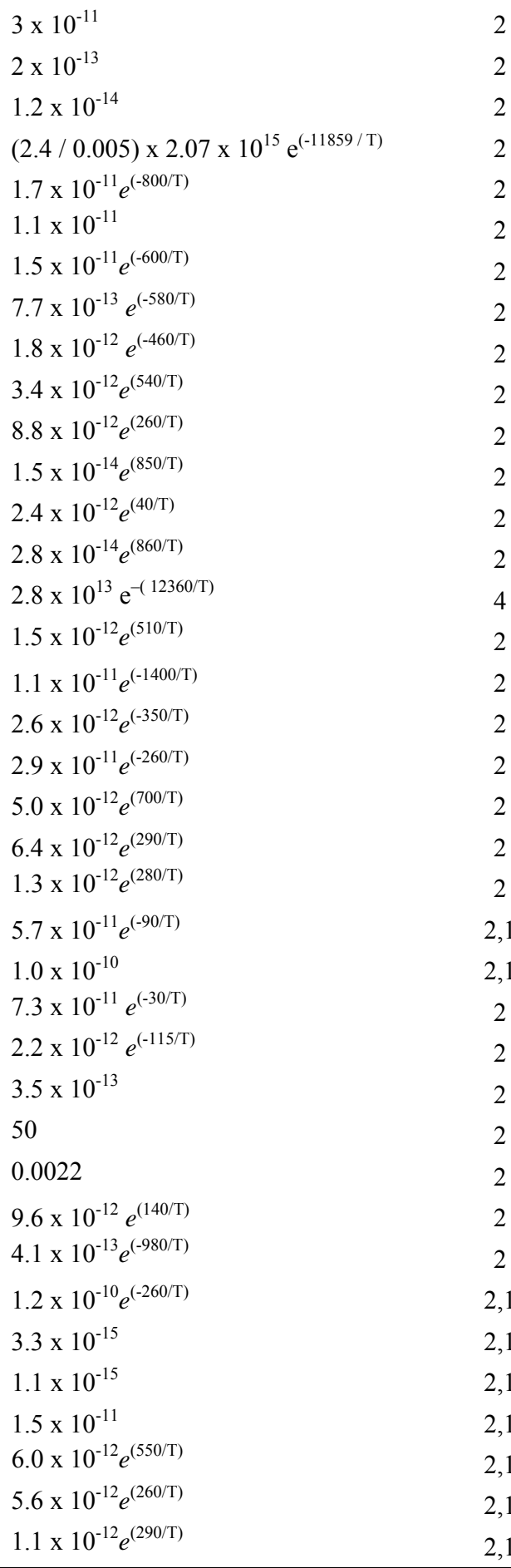

\section{Recombination Reactions}

$\begin{array}{ll}\text { 1. } & \left.\mathrm{O}^{1} \mathrm{D}\right)+\mathrm{N}_{2}(+\mathrm{M}) \rightarrow \mathrm{N}_{2} \mathrm{O}(+\mathrm{M}) \\ \text { 2. } & \mathrm{HO}_{2}+\mathrm{HO}_{2}(+\mathrm{M}) \rightarrow \mathrm{H}_{2} \mathrm{O}_{2}(+\mathrm{M}) \\ \text { 3. } & \mathrm{H}+\mathrm{O}_{2}(+\mathrm{M}) \rightarrow \mathrm{HO}_{2}(+\mathrm{M}) \\ \text { 4. } & \mathrm{O}_{2}+\mathrm{O}\left({ }^{3} \mathrm{P}\right) \rightarrow \mathrm{O}_{3} \\ \text { 5. } & \mathrm{NO}_{2}+\mathrm{OH} \rightarrow \mathrm{HNO}_{3}\end{array}$

$[\mathrm{M}] \times 3.5 \times 10^{-37} \times(\mathrm{T} / 300)^{-0.6}$

$[\mathrm{M}] \times 1.7 \times 10^{-33} \mathrm{e}^{(1000 / \mathrm{T})}$

$\mathrm{k}_{0}=5.7 \times 10^{-32} \times(\mathrm{T} / 300)^{-1.6}$ 2

$\mathrm{k}_{\infty}=7.5 \times 10^{-11}$

$[\mathrm{M}] \times 6 \times 10^{-34} \times(\mathrm{T} / 300)^{-2.3}$

$\mathrm{k}_{0}=2.5 \times 10^{-30} \times(\mathrm{T} / 300)^{-4.4}$

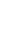

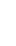
2 2

2

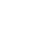

2




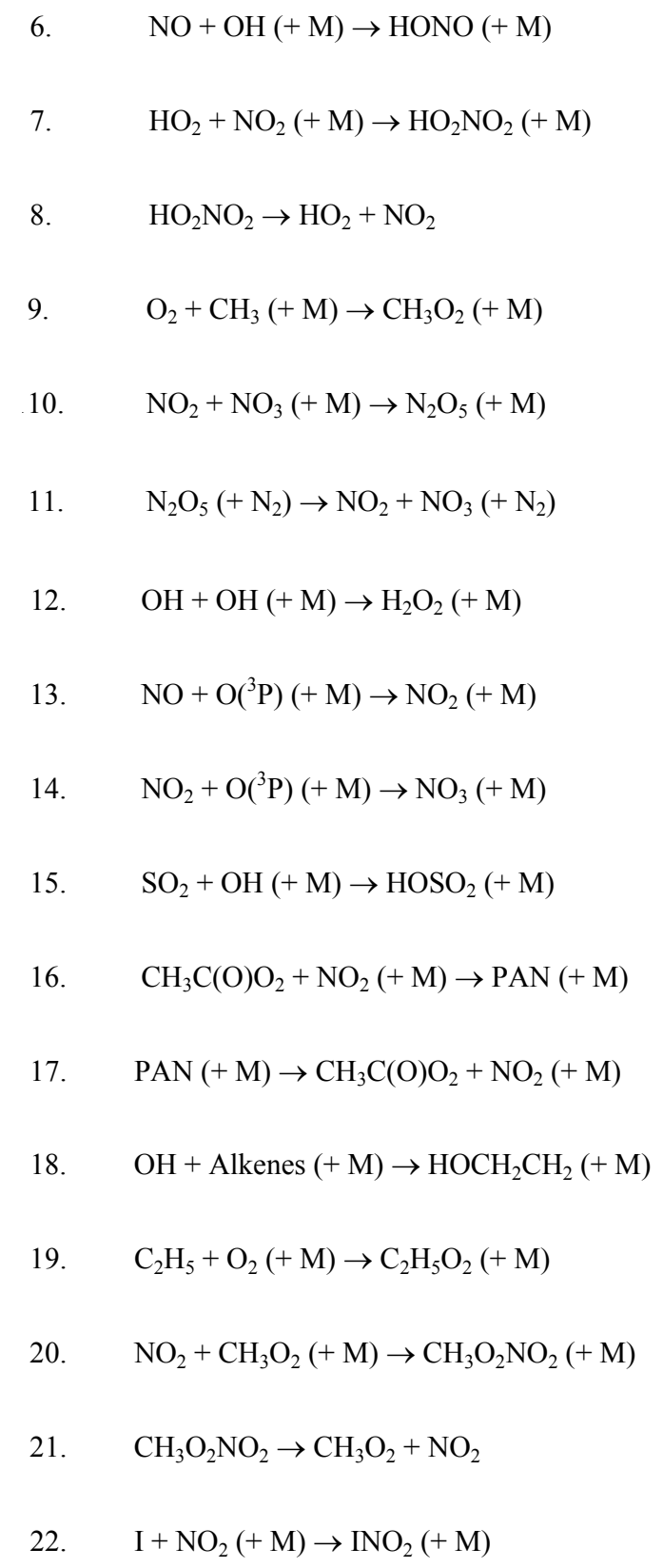

23. $\mathrm{IO}+\mathrm{NO}_{2}(+\mathrm{M}) \rightarrow \mathrm{IONO}_{2}(+\mathrm{M})$

24. $\mathrm{Br}+\mathrm{NO}_{2}+\mathrm{M} \rightarrow \mathrm{BrNO}_{2}$

25. $\mathrm{BrO}+\mathrm{NO}_{2}+\mathrm{M} \rightarrow \mathrm{BrONO}_{2}$

25. $\mathrm{ClO}+\mathrm{NO}_{2}+\mathrm{M} \rightarrow \mathrm{ClONO}_{2}$

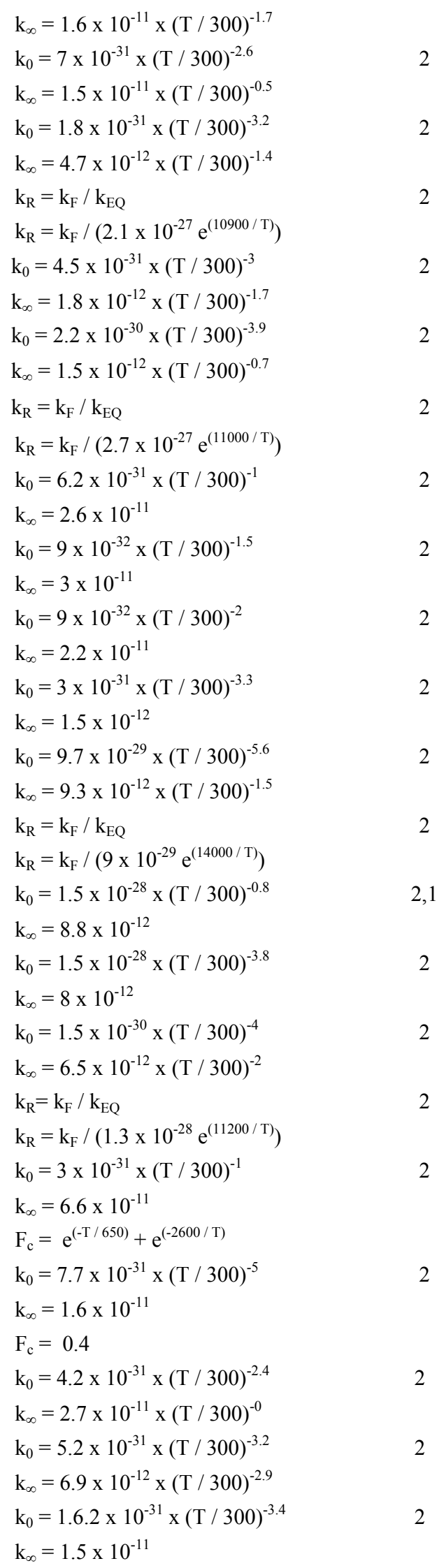




\begin{tabular}{|c|c|c|}
\hline & Photochemical Reactions & References \\
\hline $\mathrm{J} 1$. & $\mathrm{O}_{3}+h v \rightarrow \mathrm{O}_{2}+\mathrm{O}\left({ }^{1} \mathrm{D}\right)$ & $2,1, \mathrm{c}$ \\
\hline $\mathrm{J} 2$. & $\mathrm{H}_{2} \mathrm{O}_{2}+h v \rightarrow 2 \mathrm{OH}$ & $2,1, \mathrm{c}$ \\
\hline $\mathrm{J} 3$. & $\mathrm{HNO}_{3}+h v \rightarrow \mathrm{OH}+\mathrm{NO}_{2}$ & $2,1, \mathrm{c}$ \\
\hline $\mathrm{J} 4$. & $\mathrm{HO}_{2} \mathrm{NO}_{2}+h v \rightarrow \mathrm{OH}+\mathrm{NO}_{3}$ & $2,1, \mathrm{c}$ \\
\hline $\mathrm{J} 5$. & $\mathrm{HONO}+h v \rightarrow \mathrm{OH}+\mathrm{NO}$ & $2,1, \mathrm{c}$ \\
\hline J6. & $\mathrm{CH}_{3} \mathrm{OOH}+h v \rightarrow \mathrm{CH}_{3} \mathrm{O}+\mathrm{OH}$ & $2,1, \mathrm{c}$ \\
\hline $\mathrm{J} 7$. & $\mathrm{CH}_{2} \mathrm{O}+h v \rightarrow \mathrm{HCO}+\mathrm{H}$ & $2,1, \mathrm{c}$ \\
\hline $\mathrm{J} 8$. & $\mathrm{CH}_{2} \mathrm{O}+h v \rightarrow \mathrm{CO}+\mathrm{H}_{2}$ & $2,1, \mathrm{c}$ \\
\hline J9. & $\mathrm{NO}_{2}+h v \rightarrow \mathrm{NO}+\mathrm{O}$ & $2,1, \mathrm{c}$ \\
\hline $\mathrm{J} 10$. & $\mathrm{NO}_{3}+h v \rightarrow \mathrm{NO}_{2}+\mathrm{O}$ & $2,1, \mathrm{c}$ \\
\hline $\mathrm{J} 11$. & $\mathrm{N}_{2} \mathrm{O}_{5}+h v \rightarrow \mathrm{NO}_{2}+\mathrm{NO}_{3}$ & $2,1, \mathrm{c}$ \\
\hline $\mathrm{J} 12$. & $\mathrm{C}_{2} \mathrm{H}_{5} \mathrm{O}_{2} \mathrm{H}+h v \rightarrow \mathrm{OH}+\mathrm{C}_{2} \mathrm{H}_{5} \mathrm{O}$ & $2,1, \mathrm{c}$ \\
\hline $\mathrm{J} 13$. & $\mathrm{CH}_{3} \mathrm{CHO}+h v \rightarrow \mathrm{CH}_{3}+\mathrm{HCO}$ & $2,1, \mathrm{c}$ \\
\hline $\mathrm{J} 15$. & PAN $\left(\mathrm{CH} 3 \mathrm{C}(\mathrm{O}) \mathrm{O}_{2} \mathrm{NO}_{2}\right)+h v \rightarrow \mathrm{CH}_{3} \mathrm{C}(\mathrm{O}) \mathrm{O}_{2}+\mathrm{NO}_{2}$ & $2,1, \mathrm{c}$ \\
\hline $\mathrm{J} 16$. & $\mathrm{NO}_{3}+h v \rightarrow \mathrm{NO}+\mathrm{O}_{2}$ & $2,1, \mathrm{c}$ \\
\hline $\mathrm{J} 17$. & $\mathrm{CH}_{3} \mathrm{I}+h v \rightarrow \mathrm{CH}_{3}+\mathrm{I}$ & $2,1, \mathrm{c}$ \\
\hline $\mathrm{J} 18$. & $\mathrm{CH}_{2} \mathrm{I}_{2}+h v \rightarrow \mathrm{CH}_{2} \mathrm{I}+\mathrm{I} \rightarrow \mathrm{CH}_{2}+2 \mathrm{I}$ & $2,1, \mathrm{c}$ \\
\hline J19. & $\mathrm{CH}_{2} \mathrm{IBr}+h v \rightarrow \mathrm{CH}_{2} \mathrm{Br}+\mathrm{I}$ & $2,1, \mathrm{c}$ \\
\hline $\mathrm{J} 20$. & $\mathrm{I}_{2}+h v \rightarrow 2 \mathrm{I}$ & $2,1, \mathrm{c}$ \\
\hline $\mathrm{J} 21$. & $\mathrm{INO}_{2}+h v \rightarrow \mathrm{I}+\mathrm{NO}_{2} / \mathrm{IO}+\mathrm{NO}$ & $2,1, \mathrm{c}$ \\
\hline $\mathrm{J} 22$. & $\mathrm{IO}+h v \rightarrow \mathrm{I}+\mathrm{O}$ & $2,1, \mathrm{c}$ \\
\hline $\mathrm{J} 23$. & $\mathrm{OIO}+h v \rightarrow \mathrm{I}+\mathrm{O}_{2}$ & $2,1, \mathrm{c}$ \\
\hline $\mathrm{J} 24$. & $\mathrm{IONO}_{2}+h v \rightarrow \mathrm{I}+\mathrm{NO}_{3}$ & $2,1, \mathrm{c}$ \\
\hline $\mathrm{J} 25$. & $\mathrm{HOI}+h v \rightarrow \mathrm{I}+\mathrm{OH}$ & $2,1, \mathrm{c}$ \\
\hline $\mathrm{J} 26$. & $\mathrm{BrO}+h v \rightarrow \mathrm{Br}+\mathrm{O}$ & $2,1, \mathrm{c}$ \\
\hline $\mathrm{J} 27$. & $\mathrm{Br}_{2}+h v \rightarrow 2 \mathrm{Br}$ & $2,1, \mathrm{c}$ \\
\hline $\mathrm{J} 28$. & $\mathrm{IBr}+h v \rightarrow \mathrm{Br}+\mathrm{I}$ & $2,1, \mathrm{c}$ \\
\hline $\mathrm{J} 29$. & $\mathrm{BrCl}+h v \rightarrow \mathrm{Br}+\mathrm{Cl}$ & $2,1, \mathrm{c}$ \\
\hline $\mathrm{J} 30$. & $\mathrm{BrNO}_{2}+h v \rightarrow \mathrm{Br}+\mathrm{NO}_{2}$ & $2,1, \mathrm{c}$ \\
\hline $\mathrm{J} 31$. & $\mathrm{BrONO}_{2}+h v \rightarrow 0.7\left(\mathrm{BrO}+\mathrm{NO}_{2}\right) / 0.3\left(\mathrm{Br}+\mathrm{NO}_{3}\right)$ & $2,1, \mathrm{c}$ \\
\hline $\mathrm{J} 32$. & $\mathrm{HOBr}+h v \rightarrow \mathrm{Br}+\mathrm{OH}$ & $2,1, \mathrm{c}$ \\
\hline $\mathrm{J} 33$. & $\mathrm{Cl}_{2}+h v \rightarrow 2 \mathrm{Cl}$ & $2,1, \mathrm{c}$ \\
\hline $\mathrm{J} 34$. & $\mathrm{ICl}+h v \rightarrow \mathrm{I}+\mathrm{Cl}$ & $2,1, \mathrm{c}$ \\
\hline $\mathrm{J} 35$. & $\mathrm{ClO}+h v \rightarrow \mathrm{Cl}+\mathrm{O}$ & $2,1, \mathrm{c}$ \\
\hline $\mathrm{J} 36$. & $\mathrm{HOCl}+h v \rightarrow \mathrm{Cl}+\mathrm{OH}$ & $2,1, \mathrm{c}$ \\
\hline $\mathrm{J} 37$. & $\mathrm{ClNO}_{2}+h v \rightarrow \mathrm{Cl}+\mathrm{NO}_{2}$ & $2,1, \mathrm{c}$ \\
\hline $\mathrm{J} 38$. & $\mathrm{ClONO}_{2}+h v \rightarrow 0.9\left(\mathrm{Cl}+\mathrm{NO}_{3}\right) / 0.1\left(\mathrm{ClO}+\mathrm{NO}_{2}\right)$ & $2,1, \mathrm{c}$ \\
\hline
\end{tabular}




\begin{tabular}{|c|c|c|}
\hline Species & Deposition velocities, $\mathrm{cm} \mathrm{s}^{-1}$ & References \\
\hline HOI & 1.0 & 5 \\
\hline $\mathrm{HOBr}$ & 1.0 & $\mathrm{~d}$ \\
\hline $\mathrm{HOCl}$ & 1.0 & d \\
\hline $\mathrm{HBr}$ & 2.0 & d \\
\hline $\mathrm{HCl}$ & 2.0 & d \\
\hline $\mathrm{HI}$ & 1.0 & 5 \\
\hline $\mathrm{BrONO}_{2}$ & 1.0 & d \\
\hline $\mathrm{IONO}_{2}$ & 1.0 & 5 \\
\hline $\mathrm{ClONO}_{2}$ & 1.0 & $\mathrm{~d}$ \\
\hline $\mathrm{INO}_{2}$ & 1.0 & d \\
\hline
\end{tabular}

${ }^{a}$ Units: unimolecular reactions, $\mathrm{s}^{-1}$; photolysis rate constants, $\mathrm{s}^{-1}$; bimolecular reactions, $\mathrm{cm}^{3}$ molecule $\mathrm{e}^{-1} \mathrm{~s}^{-1}$; termolecular reactions, $\mathrm{cm}^{6}$ molecule $\mathrm{e}^{-2} \mathrm{~s}^{-1}$, calculated using the formalism of Sander et al. (2006), where $k$ $=\left(\left(k_{\mathrm{o}}[\mathrm{M}] /\left(1+k_{\mathrm{o}}[\mathrm{M}] / k_{\infty}\right)\right) \times F_{\mathrm{c}}{ }^{n}\right), F_{\mathrm{c}}=0.6$ (unless otherwise noted) and $n=\left(1+\left(\log _{10}\left(k_{\mathrm{o}}[\mathrm{M}] / k_{\infty}\right)\right)^{2}\right)^{-1}$.

bet as upper limit.

cabsorption cross-sections taken from Atkinson et al., 2000.

${ }^{\mathrm{d}}$ deposition velocities estimated.

Table 2. QLL Reactions and Rate Constants

\begin{tabular}{|c|c|c|c|}
\hline$\#$ & Reactions & Rate Constants & References \\
\hline 1. & $\mathrm{HOI}+\mathrm{I}^{-}+\mathrm{H}^{+} \rightarrow \mathrm{I}_{2}+\mathrm{H}_{2} \mathrm{O}$ & $4.4 \times 10^{12} \mathrm{M}^{-2} \mathrm{~s}^{-1} /(\text { volumetric })^{2}$ & 6 \\
\hline 2. & $\mathrm{I}_{2}+\mathrm{H}_{2} \mathrm{O} \rightarrow \mathrm{HOI}+\mathrm{I}^{-}+\mathrm{H}^{+}$ & $0 \mathrm{~s}^{-1}$ & \\
\hline 3. & $\mathrm{HOI}+\mathrm{Br}^{-}+\mathrm{H}^{+} \rightarrow \mathrm{IBr}+\mathrm{H}_{2} \mathrm{O}$ & $3.3 \times 10^{12} \mathrm{M}^{-2} \mathrm{~s}^{-1} /(\text { volumetric })^{2}$ & 7 \\
\hline 4. & $\mathrm{IBr}+\mathrm{H}_{2} \mathrm{O} \rightarrow \mathrm{HOI}+\mathrm{Br}^{-}+\mathrm{H}^{+}$ & $8.0 \times 10^{5} \mathrm{~s}^{-1}$ & 7 \\
\hline 5. & $\mathrm{HOI}+\mathrm{Cl}^{-}+\mathrm{H}^{+} \rightarrow \mathrm{ICl}+\mathrm{H}_{2} \mathrm{O}$ & $2.9 \times 10^{10} \mathrm{M}^{-2} \mathrm{~s}^{-1} /(\text { volumetric })^{2}$ & 8 \\
\hline 6. & $\mathrm{ICl}+\mathrm{H}_{2} \mathrm{O} \rightarrow \mathrm{HOI}+\mathrm{Cl}^{-}+\mathrm{H}^{+}$ & $2.4 \times 10^{6} \mathrm{~s}^{-1}$ & 8 \\
\hline 7. & $\mathrm{HOBr}+\mathrm{Br}^{-}+\mathrm{H}^{+} \rightarrow \mathrm{Br}_{2}+\mathrm{H}_{2} \mathrm{O}$ & $1.6 \times 10^{10} \mathrm{M}^{-2} \mathrm{~s}^{-1} /(\text { volumetric })^{2}$ & 9 \\
\hline 8. & $\mathrm{Br}_{2}+\mathrm{H}_{2} \mathrm{O} \rightarrow \mathrm{HOBr}+\mathrm{Br}^{-}+\mathrm{H}^{+}$ & $9.7 \times 10^{1} \mathrm{~s}^{-1}$ & 9 \\
\hline 9. & $\mathrm{HOBr}+\mathrm{Cl}^{-}+\mathrm{H}^{+} \rightarrow \mathrm{BrCl}+\mathrm{H}_{2} \mathrm{O}$ & $5.6 \times 10^{9} \mathrm{M}^{-2} \mathrm{~s}^{-1} /(\text { volumetric })^{2}$ & 10 \\
\hline 10. & $\mathrm{BrCl}+\mathrm{H}_{2} \mathrm{O} \rightarrow \mathrm{HOBr}+\mathrm{Cl}^{-}+\mathrm{H}^{+}$ & $1.0 \times 10^{5} \mathrm{~s}^{-1}$ & 10 \\
\hline 11. & $\mathrm{BrCl}+\mathrm{Br}^{-} \rightarrow \mathrm{Br}_{2} \mathrm{Cl}^{-}$ & $5.0 \times 10^{9} \mathrm{M}^{-1} \mathrm{~s}^{-1} /($ volumetric $)$ & 10 \\
\hline 12. & $\mathrm{Br}_{2} \mathrm{Cl}^{-} \rightarrow \mathrm{BrCl}+\mathrm{Br}^{-}$ & $2.8 \times 10^{5} \mathrm{~s}^{-1}$ & 10 \\
\hline 13. & $\mathrm{Br}_{2} \mathrm{Cl}^{-} \rightarrow \mathrm{Br}_{2}+\mathrm{Cl}^{-}$ & $3.8 \times 10^{9} \mathrm{~s}^{-1}$ & 10 \\
\hline 14. & $\mathrm{Br}_{2}+\mathrm{Cl}^{-} \rightarrow \mathrm{Br}_{2} \mathrm{Cl}^{-}$ & $5.0 \times 10^{9} \mathrm{M}^{-1} \mathrm{~s}^{-1} /($ volumetric $)$ & 10 \\
\hline 15. & $\mathrm{BrCl}+\mathrm{Cl}^{-} \rightarrow \mathrm{BrCl}_{2}^{-}$ & $5.0 \times 10^{9} \mathrm{M}^{-1} \mathrm{~s}^{-1} /($ volumetric $)$ & 11 \\
\hline 16. & $\mathrm{BrCl}_{2}^{-} \rightarrow \mathrm{BrCl}+\mathrm{Cl}^{-}$ & $1.3 \times 10^{9} \mathrm{~s}^{-1}$ & 11 \\
\hline 17. & $\mathrm{HOBr}+\mathrm{I}^{-} \rightarrow \mathrm{IBr}+\mathrm{OH}^{-}$ & $5.0 \times 10^{9} \mathrm{M}^{-1} \mathrm{~s}^{-1} /($ volumetric $)$ & 12 \\
\hline 18. & $\mathrm{HOCl}+\mathrm{Cl}^{-}+\mathrm{H}^{+} \rightarrow \mathrm{Cl}_{2}+\mathrm{H}_{2} \mathrm{O}$ & $2.2 \times 10^{4} \mathrm{e}^{(-3508 / \mathrm{T})} \mathrm{M}^{-2} \mathrm{~s}^{-1} /(\text { volumetric })^{2}$ & 13 \\
\hline
\end{tabular}


19. $\mathrm{Cl}_{2}+\mathrm{H}_{2} \mathrm{O} \rightarrow \mathrm{HOCl}+\mathrm{Cl}^{-}+\mathrm{H}^{+}$

20. $\mathrm{HOCl}+\mathrm{Br}^{-}+\mathrm{H}^{+} \rightarrow \mathrm{BrCl}+\mathrm{H}_{2} \mathrm{O}$

21. $\mathrm{BrCl}+\mathrm{H}_{2} \mathrm{O} \rightarrow \mathrm{HOCl}+\mathrm{Br}^{-}+\mathrm{H}^{+}$

22. $\mathrm{HOCl}+\mathrm{I}^{-}+\mathrm{H}^{+} \rightarrow \mathrm{ICl}+\mathrm{H}_{2} \mathrm{O}$

23. $\mathrm{ICl}+\mathrm{H}_{2} \mathrm{O} \rightarrow \mathrm{HOCl}+\mathrm{I}^{-}+\mathrm{H}^{+}$
$2.2 \times 10^{1} \mathrm{e}^{(-8012 / \mathrm{T})} \mathrm{s}^{-1}$

13

$3.5 \times 10^{11} \mathrm{M}^{-2} \mathrm{~s}^{-1} /(\text { volumetric })^{2}$

$0 \mathrm{~s}^{-1}$

$3.9 \times 10^{-14} \mathrm{e}^{(-900 / \mathrm{T})} \mathrm{M}^{-2} \mathrm{~s}^{-1} /(\text { volumetric })^{2}$

$0 \mathrm{~s}^{-1}$

Table 3. Henry Constants

\begin{tabular}{|c|c|c|}
\hline Species & Henry Constants & References \\
\hline IO & $4.5 \times 10^{2} \mathrm{e}^{(5862(1 / \mathrm{T}-1 / \mathrm{To}))} \mathrm{M} \mathrm{atm}^{-1}$ & 16 \\
\hline HOI & $4.5 \times 10^{2} \mathrm{e}^{(5862(1 / \mathrm{T}-1 / \mathrm{To}))} \mathrm{M} \mathrm{atm}^{-1}$ & 16,17 \\
\hline $\mathrm{I}_{2}$ & $3.0 \times 10^{0} \mathrm{e}^{(4431(1 / \mathrm{T}-1 / \mathrm{To}))} \mathrm{M} \mathrm{atm}^{-1}$ & 18 \\
\hline $\mathrm{ICl}$ & $1.1 \times 10^{2} \mathrm{e}^{(5600(1 / \mathrm{T}-1 / \mathrm{To}))} \mathrm{M} \mathrm{atm}^{-1}$ & 19 \\
\hline $\mathrm{IBr}$ & $2.4 \times 10^{1} \mathrm{e}^{(5600(1 / \mathrm{T}-1 / \mathrm{To}))} \mathrm{M} \mathrm{atm}^{-1}$ & 20 \\
\hline $\mathrm{HOBr}$ & $9.3 \times 10^{1} \mathrm{e}^{(5862(1 / \mathrm{T}-1 / \mathrm{To}))} \mathrm{M} \mathrm{atm}^{-1}$ & 16,20 \\
\hline $\mathrm{Br}_{2}$ & $7.6 \times 10^{-1} \mathrm{e}^{(4094(1 / \mathrm{T}-1 / \mathrm{To}))} \mathrm{M} \mathrm{atm}^{-1}$ & 21 \\
\hline $\mathrm{BrCl}$ & $9.4 \times 10^{-1} \mathrm{e}^{(5600(1 / \mathrm{T}-1 / \mathrm{To}))} \mathrm{M} \mathrm{atm}^{-1}$ & 17 \\
\hline $\mathrm{HOCl}$ & $6.7 \times 10^{2} \mathrm{e}^{(5862(1 / \mathrm{T}-1 / \mathrm{To}))} \mathrm{M} \mathrm{atm}^{-1}$ & 17 \\
\hline $\mathrm{Cl}_{2}$ & $9.1 \times 10-^{2} \mathrm{e}^{(2500(1 / \mathrm{T}-1 / \mathrm{To}))} \mathrm{M} \mathrm{atm}^{-1}$ & 22 \\
\hline
\end{tabular}




\section{References}

1. Atkinson, R., Baulch, D. L., and Cox, R. A.: J. Phys. Chem. Ref. Data, 29, 2005.

2. Sander, S. P., Friedl, R. R., Golden, D. M., Kurylo, M. J., Moortgat, G. K., KellerRudek, H., Wine, P. H., Ravishankara, A. R., Kolb, C. E., Molina, M. J., FinlaysonPitts, B. J., Huie, R. E., and Orkin, V. L.: Chemical kinetics and photochemical data for use in atmospheric studies, JPL-NASA, 2006.

3. Martin, J. C. G., Spietz, P., Burrows, J. P.: Spectroscopic studies of the $\mathrm{I}_{2} / \mathrm{O}_{3}$ photochemistry - Part 1: Determination of the absolute absorption cross sections of iodine oxides of atmospheric relevance, J. Photochemistry and Photobiology A., $176,15-38,2005$.

4. Orlando, J. \& Tyndall, G. S.: Rate coefficients for the thermal decomposition of $\mathrm{BrONO}_{2}$ and the heat of formation of $\mathrm{BrONO}_{2}$, J. Phys. Chem. A., 100, 1939819405, 1996.

5. Jenkin, M. E.: Environ. and Energy Rep. AEA EE-0405, Harwell Lab., Oxfordshire, England, 1992.

6. Eigen, M. \& Kustin, K.: Kinetics of halogen hydrolysis, J. Am. Chem. Soc., 84, 1355-1361 (1962).

7. Troy, R. C., Kelley, M. D., Nagy, J. C., and Margerum, D. W.: Nonmetal redox kinetics - iodine monobromide reaction with iodide-ion and the hydrolysis of IBr, Inorg. Chem., 30, 4838- 4845, 1991.

8. Wang, Y. L., Nagy, J. C., and Margerum, D. W.: Kinetics of hydrolysis of iodine monochloride measured by the pulsed-accelerator-flow method, J. Am. Chem. Soc., 111, 7838- 7844, 1989.

9. Beckwith, R. C., Wang, T. X., Margerum, D. W.: Equilibrium and kinetics of bromine hydrolysis, Inorg. Chem., 35, 995-1000, 1996.

10. Wang, T. X., Kelly, M. D., Cooper, J. N., Beckwith, R. C., and Margerum, D. W.: Equilibrium, kinetic, and UV-spectral characteristics of aqueous bromine chloride, bromine, and chlorine species, Inorg. Chem., 33, 5872-5878, 1994.

11. Liu, Q. \& Margerum, D. W.: Equilibrium and kinetics of bromine chloride hydrolysis, Environ. Sci. Technol., 35, 1127-1133, 2001.

12. Troy, R. C. \& Margerum, D. W.: Nonmetal redox kinetics - hypobromite and hypobromous acid reactions with iodide and with sulfite and the hydrolysis of bromosulfate, Inorg. Chem., 30, 3538-3543, 1991.

13. Wang, T. X. \& Margerum, D. W.: Kinetics of reversible chlorine hydrolysis: temperature dependence and general- acid/based-assisted mechanisms, Inorg. Chem., 33, 1050-1055, 1994.

14. Kumar, K. \& Margerum, D. W.: Kinetics and mechanism of general-acid-assisted oxidation of bromide by hypochloriteand hypochlorous acid, Inorg. Chem., 26, 2706-2711, 1987. 
15. Nagy, J. C., Kumar, K., and Margerum, D. W.: Non-metal Redox Kinetics Oxidation of iodide by hypochlorous acid and by nitrogen trichloride measured by the pulsed-accelerated-flow method, Inorg. Chem., 27, 2773-2780, 1988.

16. Huthwelker, T., Peter, Th., Luo, B. P., Clegg, S. L., Carslaw, K. S., and Brimblecombe, P.: Solubility of HOCL in water and aqueous $\mathrm{H}_{2} \mathrm{SO}_{4}$ to stratospheric temperatures, J. Atmos. Chem., 21, 81-95, 1995.

17. Chatfield, R. B. \& Crutzen, P. J.: Are there interactions of iodine and sulfur species in marine air photochemistry, J. Geophys. Res., 95D, 22319-22341, 1990.

18. Palmer, D. A., Ramette, R. W., and Mesmer, R. E.: The hydrolysis of iodine equilibria at high-temperatures, J. Nucl. Mater., 130, 280-286, 1985.

19. Bartlett, W. P. \& Margerum, D. W.: Temperature dependencies of the Henry's Law constant and the aqueous phase dissociation constant of bromine chloride, Environ. Sci. Technol., 33, 3410-3414, 1999.

20. Vogt, R., Crutzen, P. J., and Sander, R.: A mechanism for halogen release from seasalt aerosol in the remote marine boundary layer, Nature, 383, 327-330, 1996.

21. Dean, J. A.: Lange's Handbook of Chemistry, McGraw-Hill, Inc., 1992.

22. Wilhelm, E., Battino, R., and Wilcock, R. J.: Low-pressure solubility of gases in liquid water, Chem. Rev., 77, 219-262, 1977. 